VÉRONIQUE BESSIÈRE, ÉRIC STÉPHANY

Université de Montpellier, IAE

\title{
PETER WIRTZ
}

Université Jean Moulin Lyon 3, iaelyon school of management

\section{Gouvernance et financement des firmes entrepreneuriales}

\section{Nouvelles perspectives}

$\mathrm{L}$

e marché de la finance entrepreneuriale a considérablement évolué au cours de la dernière décennie

(Wallmeroth et al., 2018). De nouveaux acteurs ont fait leur apparition (Bellavitis et al., 2017), dont notamment les différentes formes de plateformes de crowdfunding (Bessière et Stéphany, 2017), et d'anciens acteurs ont fait évoluer leur mode d'organisation traditionnel, tels que les business angels, dont les groupes tendent vers un niveau de professionnalisation plus élevé que par le passé (Bonnet et al., 2021). Les firmes entrepreneuriales innovantes ont comme principales caractéristiques d'évoluer dans un environnement hautement incertain et d'être affectées par un degré d'asymétrie informationnelle très élevé. Elles sont confrontées à «l'hypothèque de la nouveauté » ou liability of newness (Stichcomb, 1965). Cela implique, pour les acteurs de la 
finance entrepreneuriale, la nécessité de disposer d'aptitudes particulières, en matière à la fois cognitive et informationnelle. Reconnaître un potentiel de création de valeur dans un projet innovant, où le marché reste encore à créer, par exemple, n'a rien d'évident et, même si le potentiel est reconnu par un ensemble d'acteurs, la transformation en entreprise viable et la gestion de la croissance des activités nécessitent des connaissances et des compétences particulières, dont les équipes entrepreneuriales ne disposent pas nécessairement au départ (Stinchcombe, 1965 ; Hambrick et Crozier, 1985). Par ailleurs, compte tenu de leur faible antériorité et du manque d'outils et de procédures dans les domaines de la production et de la transmission de l'information, les jeunes firmes entrepreneuriales posent à leurs investisseurs des défis particulièrement aigus en matière de sélection adverse et de risque moral.

La gestion de la relation entre les acteurs de la finance entrepreneuriale et les entrepreneurs implique typiquement les mécanismes de gouvernance (Lang et Wirtz, 2021; Wirtz, 2011), et chaque forme de finance entrepreneuriale affiche ses spécificités en la matière ( $c f$. Bessière et Stéphany, 2014, 2015 ; Cumming et al., 2021 pour un exemple récent de programme de recherche sur la gouvernance dans un contexte de crowdfunding). Toujours est-il que la recherche sur la finance et la gouvernance entrepreneuriales a pour l'essentiel été menée acteur par acteur (Cumming et Vismara, 2017 ; Wallmeroth et al., 2018), et rares sont encore les travaux qui procèdent d'une vision multi-acteurs, qui débouchent sur la compréhension de véritables trajectoires de financement
(Bessière et al., 2020) et, partant, des évolutions complexes et dynamiques des systèmes de gouvernance liés.

Ce dossier spécial est consacré à ces problématiques récentes en matière de financement et de gouvernance des firmes entrepreneuriales. Il rassemble cinq contributions originales de chercheurs français qui adoptent des perspectives et des méthodes multiples, mais complémentaires, pour étudier ce phénomène complexe.

En matière d'acteurs, deux articles s'intéressent à l'Equity Based Crowdfunding (ECF). Devant la multitude des plateformes concernant ce mode de financement en pleine émergence, Bouglet, Garmilis et Joffre (Bouglet et al., 2021) développent une typologie, fondée sur l'observation empirique, des divers modèles de gouvernance adoptés par les plateformes françaises. Bouaiss et Girard-Guerraud (2021) étudient les déterminants de la faillite des entreprises financées par equity crowdfunding et concluent notamment que certains éléments du capital humain peuvent servir aux investisseurs de la foule de signal de qualité, diminuant d'autant le risque d'une faillite ultérieure.

Burkhardt-Bourgeois et Bonnet (2021), quant à eux, étudient la gouvernance cognitive et disciplinaire exercée par des sociétés de capital investissement dans le contexte de processus de build-up et son lien avec la croissance.

Vilanova et Vitanova (2021) examinent la perception du narcissisme et de l'humilité des entrepreneurs par différents types d'investisseurs en capital, dont notamment les capital-risqueurs et les business angels. Ils étudient plus particulièrement le lien entre ces caractéristiques psychologiques et la sélection par les 
investisseurs des projets portés par ces entrepreneurs.

Tarillon (2021), enfin, s'intéresse aux évolutions des fonctions de la gouvernance (cognitive $v s$. disciplinaire) exercée par les actionnaires et leur impact sur la croissance des firmes. Là où beaucoup de recherches en matière de finance entrepreneuriale adoptent la perspective de l'investisseur (venture capital, business angel ou foule), l'originalité de l'approche de Tarillon consiste à interroger la perception du dirigeant-entrepreneur de l'apport des actionnaires et de la relation entretenue avec ces derniers. $\mathrm{Vu}$ sous cet angle, le financement et la gouvernance des firmes entrepreneuriales ne sont pas simplement une affaire de sélection d'une firme par un ou plusieurs acteurs de la finance entrepreneuriale, mais aussi une possible co-construction d'une véritable trajectoire de financement (Bessière et al., 2020) dont le dirigeant est le moteur, à l'intérieur d'un marché de la finance entrepreneuriale en pleine mutation et de plus en plus complexe.

La variété des acteurs et des objets abordés par les articles qui forment ce dossier spécial coïncide avec l'hétérogénéité des méthodes de recherche mobilisées. Elle couvre en effet un vaste champ de positionnements méthodologiques, comprenant la réflexion conceptuelle (Vilanova et Vitanova, 2021), l'étude de cas qualitative (Burkhardt-Bourgeois et Bonnet, 2021), ainsi que différentes approches quantitatives (Bouglet et al., 2021; Bouaiss et Girard-Guerraud, 2021 ; Tarillon, 2021). Il est à noter que la finance et la gouvernance entrepreneuriales, par la complexité de leurs objets, se prêtent particulièrement bien à l'investigation à travers des méthodes mixtes (Levasseur et al., 2021).

Sur le fond, les contributions de ce dossier montrent qu'il n'y a pas de modèle de gouvernance unique pour la gestion des relations entre acteurs de la finance entrepreneuriale et entrepreneurs. Les approches dépendent à la fois des acteurs spécifiques qui interviennent dans un cas d'espèce et de leur combinaison possible, mais aussi de la psychologie (Vilanova et Vitanova, 2021), du capital humain et des compétences des entrepreneurs (Bouaiss et Girard-Guerraud, 2021 ; Tarillon, 2021), voire du stade de développement de l'entreprise (BurkhardtBourgeois et Bonnet, 2021 ; Tarillon, 2021). Si la dimension cognitive est très présente dans le contexte de la gouvernance entrepreneuriale, on ne saurait négliger l'existence d'une composante disciplinaire, parfois forte, également (Bouaiss et Girard-Guerraud, 2021 ; Tarillon, 2021 ; Lang et Wirtz, 2021). Cela confirme que la finance entrepreneuriale est un champ d'application particulièrement pertinent d'une approche intégrée de la gouvernance, qui recourt à la fois à des explications cognitives et disciplinaires, voire comportementales (Charreaux et Wirtz, 2006).

Pour l'avenir, l'articulation précise de ces dimensions mériterait des approfondissements, comme par ailleurs la dynamique temporelle de l'interaction entre acteurs multiples (venture capital, business angel, foule, entrepreneurs) (Bessière et al., 2020 ; Cohen et Wirtz, 2021). 


\section{BIBLIOGRAPHIE}

Bellavitis C., Filatotchev I., Kamuriwo D.S. et Vanacker T. (2017). "Entrepreneurial finance: new frontiers of research and practice”, Editorial, special issue, Embracing Entrepreneurial Funding Innovations.

Bessière V., Stéphany É. et Wirtz P. (2020). "Crowdfunding, business angels, and venture capital: An exploratory study of the concept of the funding trajectory", Venture Capital, vol. 22.

Bessière V. et Stéphany É. (2014). « Le financement par crowdfunding. Quelles spécificités pour l'évaluation des entreprises ? », Revue française de gestion, vol. 40, n 242, p. 149-161.

Bessière V. et Stéphany É. (2015). « Financement et gouvernance des start-ups en equitycrowdfunding », Finance, Contrôle, Stratégie, vol. 18-4.

Bessière V. et Stéphany É. (2017). Le crowdfunding : fondements et pratiques, De Boeck, 2 éd.

Bonnet C., Capizzi V., Cohen L., Petit A. et Wirtz P. (2021). "What drives the active involvement in business angel groups? The role of angels' decision-making style, investment-specific human capital and motivations", Journal of Corporate Finance, 101944.

Bouaiss K. et Girard-Guerraud C. (2021). « Capital humain du dirigeant-entrepreneur et faillite des firmes financées par ECF », Revue française de gestion, vol. 47, ce n ${ }^{\circ}$ 299, p. 95-115.

Bouglet J., Garmilis G. et Joffre O. (2021) «Gouvernance cognitive et développement des ressources entrepreneuriales. Étude des plateformes françaises d'equity crowdfunding », Revue française de gestion, vol. 47, $\mathrm{n}^{\circ} 299$, p. 67-94.

Burkhardt-Bourgeois K. et Bonnet C. (2021). « Comment les capital-investisseurs aident-ils leurs participations à croître ? Le cas des build-up », Revue française de gestion, vol. 47, $\mathrm{n}^{\mathrm{o}} 299$, p. 117-137.

Charreaux G. et Wirtz P. (2006). Gouvernance des entreprises, Economica.

Cohen L. et Wirtz P. (2021). "Decision-making style in entrepreneurial finance and growth", Small Business Economics, à paraître.

Cumming D.J., Vanacker T. et Zahra S.A. (2021). "Equity crowdfunding and governance: Toward an integrative model and research agenda", Academy of Management Perspectives, vol. $35, \mathrm{n}^{\mathrm{o}} 1$, p. 69-95.

Cumming D.J. et Vismara S. (2017). "De-segmenting research in entrepreneurial finance", Venture Capital, vol. 19, n ${ }^{\circ}$ 1-2, p. 17-27.

Hambrick D.C. et Crozier L.M. (1985). "Stumblers and stars in the management of rapid growth", Journal of Business Venturing, vol. 1, $\mathrm{n}^{\mathrm{o}}$ 1, p. 31-45.

Lang, N.K. et Wirtz P. (2021). "Kicking off the corporate governance lifecycle: Seed funding, venture capital and the nascent board", British Journal of Management.

Levasseur L., Johan S. et Eckhardt J. (2021). "Mixed methods in venture capital research: An illustrative study and directions for future work", British Journal of Management. 
Stinchcombe A.L. (1965). Social Structure and Organizations. In Handbook of Organizations, March JG (Ed), Chicago, Rand McNally: Chicago, IL, p. 142-193.

Tarillon C. (2021). «Vers une gouvernance coercitive pour soutenir la croissance des startup ?», Revue française de gestion, vol. 47, n 299, p. 163-183.

Vilanova L. et Vitanova I. (2021). « Humilité et narcissisme des entrepreneurs. Quel impact sur les décisions des investisseurs en capital ? », Revue française de gestion, vol. 47, n 299 , p. 139-161.

Wallmeroth J., Wirtz P. et Groh A.P. (2018). "Venture capital, angel financing, and crowdfunding of entrepreneurial ventures: A literature review", Foundations and Trends in Entrepreneurship, vol. 14, $\mathrm{n}^{\mathrm{o}}$ 1, p. 1-129.

Wirtz P. (2011). "The cognitive dimension of corporate governance in fast growing entrepreneurial firms", European Management Journal, vol. 29, nº 6, p. 431-447. 